\title{
Promote severe wound healing by PVP-I dry powder spray: case series report
}

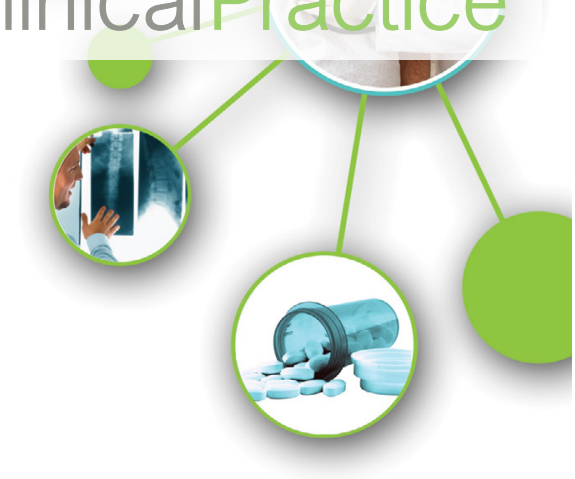

Povidone-iodine (PVP-I) is well-known for its anti-infection role in a wide range of wound types. Nowadays, appropriate use of PVP-I new formulation can absorb the exudate and reduce infection in severe wounds. The report is to record the clinical application of PVP-I dry powder spay (Betadine) as a leave-on antiseptic on four kinds of wounds. The clinical experience can provide the alternative management in dealing with these wounds in a convenient and efficient way. In the case series report, it includes four cases such as macerated wound, skin abrasion, ischemia wound and ulcer wound with the aid of PVP-I dry powder spray during February 2016 to June 2017. From the cases, continued use of PVP-I dry powder spray can significantly cease the infection and discharge, reduce the size of wound, and facilitate wound healing. Our experience also indicates PVP-I's efficacy on infection prophylaxis. Furthermore, the new formulation of PVP-I dry powder spray provides great convenience for nursing professionals during wound management.

\section{Keywords: povidone-iodine, antiseptics, exudating wounds, wound healing.}

\section{Introduction}

The wound management system in Taiwan implement patient-centered concept to fit modalities for individual situation [1]. Wound care, a continuous process which is not fully understood and complied by patients, ends up to reoccurrence or healing failure. The microenvironment of skin wounds mentioned by Scalise et al interprets that infection of wounds occurs when the dynamic equilibrium between microorganism and human immune defense is disturbed [2]. Therefore, the infection control comes the priority of wound care. Bioburden, known as bacterial critically colonization, usually impedes wound healing process, with lack of current methods to identify the biofilm existing in most of unhealable wounds [2,3]. Thomas et al. state that the application of antiseptics should be the first line to handle suspected or existed infection [4]. Ideal antiseptics enable patients to infection prophylaxis and delay the use of antibiotics which are reported with bacterial resistance. Also antiseptics with broad spectrum antimicrobial effect are recommended to prevent bioburden in the wound which may raise the occurrence of local infection, and even life-threatening systematic infection [3]. lodine primarily acts as an antimicrobial agent as free iodine oxidizes vital pathogen structures and thus deactivates microbial DNA/RNA and protein. Povidone-iodine, lodophors, contains elementiodine complexed with solubilizing agent, polyvinylyrrolidone (povidone). They form a water-soluble compound, with less irritating and good skin tolerance [5]. Free iodine released from PVP-I reservoir exhibits bactericidal activity when contacting with skin and mucous membranes. PVP-I has a broader antimicrobial spectrum than other antiseptics, rapid and potent microbicidal effect, and the ability to disrupt biofilms without the occurrence of resistance reported [5-7]. The above characteristics make PVP-I extraordinarily well for daily medical or consumer use in treating both acute and chronic wounds with infection and exudate. Furthermore, clinical efficacy of PVP-I dry powder spray not only reduces the rate of infection and discharge in acute wounds, but also exerts hemostatic effect on superficial bleeding [5].

A systematic review published in 2010 indicates that half of PVP-I trials are more than 10 years ago. Pressure ulcers and acute wounds with PVP-I use explained the reduction of bacterial load and infection rates

\author{
Hui-Mei Yang ${ }^{1}$, \\ Jiun-Ting Yeh \\ ${ }^{1}$ Department of Endocrinology and \\ Metabolism, Chang Gung Memorial \\ Hospital Linkou Branch, Taoyuan, \\ Taiwan, R.O.C \\ ${ }^{2}$ Division of Trauma, Department of \\ Plastic and Reconstructive Surgery, \\ Chang-Gung University Medical \\ Department, Chang-Gung Memorial \\ Hospital Linkou Branch, Taoyuan, \\ Taiwan, R.O.C \\ *Author for correspondence.
}

Yates1965@gmail.com.tw 
[8]. However, the study of Dry Powder Spray is even more limited [9]. Differently, the four cases can exhibit the good healing outcomes by PVP-I Dry Powder itself. Macerated wounds for some dressings might be concerned, such as pressure ulcers and venous ulcers so that periwound should be kept from excess hydration [10]. Therefore, the purpose of this report is to demonstrate the convenience and benefit of PVP-I dry powder spray which by keeping record of common difficult wound management in patients.Liberty-institutional review board (IRB) approval from CGMH was obtained for this study (\#201801129B0).

\section{Case Report}

\section{Case I: Incontinence associated- dermatitis (IAD)}

A 43-year-old male presented with IAD resulting from chronic diarrhea. The patient was $1.67 \mathrm{~m}$ tall and $36.2 \mathrm{Kg}$ body weight (BMI 12.9, underweight). He had severe postural hypotension due to diabetic autonomic neuropathy, and had five to six times of chronic diarrhea daily. Before hospitalization, a fainting and falling led to left hip fracture and following six-day long bedridden. A $19 \times 35 \times 0.1 \mathrm{~cm}$ wound with second stage injury on the buttocks was identified when admitted. Silver Sulfadiazine cream b.i.d. was applied for three days; however, the wound became deeper as $20 \times 35 \times 0.5 \mathrm{~cm}$. The CRP level $113.43 \mathrm{mg} / \mathrm{dl}$ and WBC count $8,900 / \mu \mathrm{l}$ suggested signs of infection. The nursing practitioner then employed $7.5 \%$ PVP-I cleanser to clean necrotic tissues of periwound, and followed by $0.45 \%$ PVP-I Dry powder spray to absorb exudate t.i.d. Decreased discharge was found after sixteen days of treatment and the frequency of dry powder application was reduced to b.i.d.

The size and infection of the wound hampered the surgery on hip fracture. By continued use of PVP-I dry powder spray, exudate reduction and infection control was observed without odor after five days. The wound size decreased to $10 \times 10 \times 0.1$ $\mathrm{cm}$ and the patient eventually underwent left hip hemiarthroplasty after sixteen days of hospitalization. The patient still had daily diarrhea five to six times during hospitalization but the wound ultimately healed after further two weeks with 5 bottles of PVP-I dry powder spray continuously.

\section{- Case II: Epidermal injury}

A 70-year-old male presented with a $4 \times 2.5 \times 0.1 \mathrm{~cm}$ skin injury on the right posterior neck upon removal of central venous catheter. The patient was $1.66 \mathrm{~m}$ tall, $72 \mathrm{~kg}$ body weight, and BMI 26.1 (overweight). He had a history of Diabetes for more than twenty years and prostate cancer for six years. Due to hyperglycemia and hyperosmolarity, he was admitted to hospital six days after his outpatient chemotherapy. Central venous catheterization on the right neck was carried out because he had two to three times of diarrhea daily and loss of appetite. Chemotherapy destroyed his hematopoietic cells in bone marrow, which in turn led to gradual decline of WBC counts as $700 / \mu 1$ after two weeks. Central venous catheter was removed one day later and left a $4 \times 2.5 \times 0.1$ $\mathrm{cm}$ skin injury.

No signs of healing were observed after six days treatment by Neomycin and Tyrothricin ointment, and the wound size expanded to $8 \times 8 \times 0.1 \mathrm{~cm}$. After changed to PVP-I dry powder spray q.d. for two days, the condition was greatly improved with dried and healing wound bed. Complete healing of the wound was reached after continued use of dry powder spray as leave-on antiseptic for a total of five days. After wound healed, moisture maintenance was applied to prevent itching resulted from excessive dry.

\section{Case III: Peripheral arterial disease (PAD)}

This case represents the difficulties in elderly nursing care in Taiwan, i.e., the concept of keeping an intact body after death.

A 84-year-old female was admitted to hospital due to infection of left foot and loss of appetite. She was $1.46 \mathrm{~m}$ tall, 36.5 $\mathrm{kg}$ body weight, BMI 17.1 (underweight), and had a history of type 2 diabetes for more than 7 years and dementia for 4 years. When admitted, she had a bulla, a $12 \times 8 \mathrm{~cm}$ gangrene on the dorsum of her left foot and three toes for one month.

Silver Sulfadiazine cream b.i.d. was used 
initially for wound dressing for six days but not effective. The Ankle-Brachial Index (ABI) was down to zero which suggested severe vasculopathy, so the orthopedist recommended below-knee amputation. Considering her elderly and long-term bedridden, her family was reluctant to it. Then PVP-I dry powder was then used for wound management t.i.d. after appropriate cleaning. Five minutes after each spray, the toes were separated with cotton gauze and then the food pad was covered with bandage. Seventeen days later, the infection was controlled, the wound bed turned dry, and the frequency of dressing reduced to once daily. After another week, the necrotic tissue on the left foot was $20 \times 9 \mathrm{~cm}$ in size, dry and desiccated, and without signs of infection.

\section{Case IV: lower extremity venous ulcer}

A 59-year-old male was admitted to hospital with infection on his left lower leg. The patient was $1.79 \mathrm{~m}$ tall, $106 \mathrm{~kg}$ body weight, BMI 33 (overweight), with a history of type 2 diabetes for more than twelve years, venous ulcer for seven years and brain stem stroke with bilateral hemiplegia. He complained weakness of both lower limbs and had to walk with crutches for twelve years. In addition, the wound derived from venous ulcer on left lower leg was unable to heal for four years due to work-related prolonged standing.

When admitted, the wound size was $9 \times 5 \times 0.2 \mathrm{~cm}$ with surrounding tissue reddish and swollen. Silver Sulfadiazine cream b.i.d. was applied for two days but no signs of improvement was observed. PVP-I dry powder spray was then employed b.i.d. with elastic bandage to facilitate venous reflux. After he discharged with thirty four days of continued treatment above, the size of wound had significantly decreased to $3 \times 2 \times 0.1 \mathrm{~cm}$.

\section{Discussion}

Controlling infectious wounds and preparing for clean wound beds for granulating tissue are critical for restoring the physiological phases of wound healing, and prepared for further treatment $[2,4]$.

The four severe wound care experience provides nursing practitioners an alternative way to deal with infectious or infection suspected wounds. Similarly, the wound healing was not observed with initial treatment. After the application of PVP-I dry powder, the infection was controlled (case I and III) along with decreased wound size detected over time (case I and IV) or reached complete healing (case II). Additionally, PVP-I dry powder provides less-irritant and easy cleansing efficiency to target areas and improves re-epithelialization to wounds, along with great convenience for health care providers and caregivers during wound management.

Our current experience of PVP-I echoes previous studies that iodine facilitates granulation tissue formation and shortens the time required for wound closure [5] PVP-I dry powder spray exhibited successful exudate management, providing good medium to high level exudate absorption, infection control, inflammation and pain reduction during dressing changes for diabetic patients[6,9] The performance of PVP-I was superior to both silver salfadiazine and chlorhexidine digluconate in a study of chronic leg ulcers wounds. The study also suggests PVP-I an appropriate tool for routine management of non-healable and maintenance [11]. The limitation of the report is scarce of organized study and large amount of clinical experiences. Well-designed study is necessary for clinical practice of PVP-I dry powder spray in the future. However, the description of four cases can vividly deliver practical concept to nursing practitioners. Yu-Tsung Chen et al. concludes that clinical outcome of wounds are related to the function of community healthcare and patient education [1]. With clinical efficacy and much easier care of application, PVP-I dry powder spray is beneficial to nursing practitioners, caregivers and even patients themselves.

\section{Acknowledgements}

BETADINE is a registered trademark of Mundipharma Ltds. The article preparation is supported by Mundipharma Pharmaceutical Ltd. The views expressed in this manuscript are solely from the author. This research did not receive any specific grant from funding agencies in the public, commercial, or not-forprofit sectors. Patients all provided written informed consent for publication of the case series report and the accompanying images. 


\section{References}

1. Chen YT, Chang CC, Shen JH, et al. Demonstrating a Conceptual Framework to Provide Efficient Wound Management Service for a Wound Care Center in a Tertiary Hospital. Medicine (Baltimore). 94(44), e1962 (2015).

2. Scalise A. Microenvironment and microbiology of skin wounds: The role of bacterial biofilms and related factors. Semin. Vasc. Surg. 28(3-4), 151-159 (2015).

3. Barrett S. Wound-bed preparation: a vital step in the healing process. Br. J. Nurs. 26, S24-S31 (2017). 4. Bianchi T. Recommendations for the $\mathrm{e}^{223-233}$ (2017). management of biofilm: a consensus document. J Wound Care. 25(6), 305317 (2016).

5. Lachapelle JM, Castel O, Casado AF et al. Antiseptics in the era of bacterial resistance: a focus on povidone iodine. Clin. Pract. 10(5), 579-592 (2015).

6. Bigllardi PL, Alsagoff SAL, El-Kafrawl $H Y$, et al. Povidone iodine in wound healing: A review of current concepts and practices. Int. J. Surg. 44, 260-268 (2017).

7. Bigliardi P, Langer S, Cruz JJ, et al. Ar Asian Perspective on Povidone lodine in Wound Healing. Dermatology. (2-3),
8. Vermeulen $\mathrm{H}$, Westerbos $\mathrm{SJ}$, Ubbink DT. Benefit and harm of iodine in wound care a systematic review. J. Hosp. Infect. 76(3), 191-199 (2010).

9. Tongson LS. Exudate management and antisepsis in diabetic patients with problem wounds: two case reports. Chronic Wound Care Manag. Res. 2017; 4:77-81

10. Sood A, Granick MS, Tomaselli NL. Wound Dressings and Comparative Effectiveness Data. Adv. Wound Care (New Rochelle). 3(8), 511-529 (2014).

11. Woo KY. Management of nonhealable or maintenance wounds with topical povidone iodine. Int. Wound J. 11(6), 622-626 (2014). 